\title{
A TECHINIQUE FOR FINDING MINIMAL PATHS IN SUBSPACES OF A METRIC SPACE
}

\author{
HAROLD WILLIS MILNES and S. K. HILDEBRAND
}

(Received 11 January 1973; revised 26 September 1973)

Communicatcd by E. Strzelecki

In this paper the proble $m$ of constructing an arc of minimum length joining two fixed points: $p_{1}, p_{2}$, in an arbitrary subset: $S$, of a metric space is considered. The approach taken is a departure from the classical methods of the calculus of variations in that it is topological in character, making use of the properties of sets rather than differential calculus.

The subsequent development requires the following definitions. The metric distance betwenn two points $p, q$ is denoted as $\rho(p, q)$ and the open sphere of radius: $a$, by $S_{a}(p) \equiv\{q \mid \rho(p, q)<a\}$.

$$
\text { The set: } \begin{cases}T_{a}(p) & \text { is the component containing } p \text { in } S_{a}(p) \cap S ; \\ T_{a}^{(1)}(p) \equiv T_{a}(p) \text { and } T_{a}^{(k+1)}(p)=\bigcup_{q \in T_{a}^{(k)}(p)} T_{a}(q), \quad(k=1,2, \cdots) ; \\ U_{c}(p) \equiv \bigcap_{j=1}^{\infty} T_{c / j}^{(j)}(p) ; \\ V_{c}(p) \equiv \bigcap_{\epsilon>0} U_{c+d}(p), \text { where } c \geqq 0 \text { is a constant. }\end{cases}
$$

As an illustrative example of the above definitions one may consider the plane with the line segment from $(1 / 2,-2 / 3)$ to $(1 / 2,2 / 3)$ removed. The sets $T_{1 / a}^{(a)}(0)$, $(a=1,2,3,4)$ and $U_{1}(0)$ are shown in Figure 1.

LeMMA 1. $V_{c}(p) \subset V_{c+x}(p)$ for every $x \geqq 0$.

Proof. $T_{a}(p) \subset T_{b}(p), a \leqq b$, and therefore $T_{a}^{(k)}(p) \subset T_{b}^{(k)}(p)$.

Thus,

$$
U_{c}(p)=\bigcap_{j=1}^{\infty} T_{c / j}^{(j)}(p) \subset \bigcap_{j=1}^{\infty} T_{(c+x) / j}^{(j)}(p)=U_{c+x}(p) .
$$




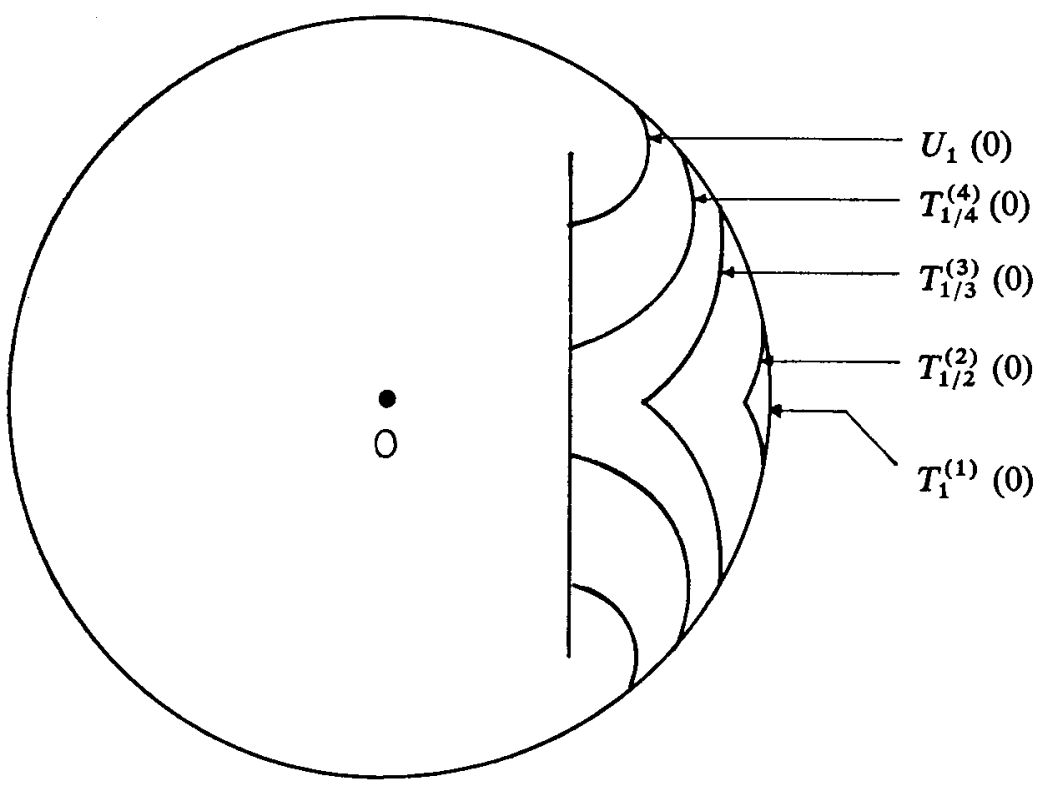

Figure 1.

Finally

$$
V_{c}(p)=\bigcap_{\varepsilon>0} U_{c+\varepsilon}(p) \subset \bigcap_{\varepsilon>0} U_{c+x+\varepsilon}(p)=V_{c+x}(p) .
$$

LEMMA 2. Suppose that $\left\{t_{i} \mid i=1,2, \cdots\right\}$ is a monotone non-increasing sequence tending to the limit $t_{0}$, and that $q \in V_{t_{i}}(p)$ for all $i$; then $q \in V_{t_{0}}(p)$.

Proof. By Lemma 1, since $V_{t_{i}+x}(p) \supset V_{t_{1}}(p)$ for all $x>0$ it follows that $q \in V_{t}(p)$ for all $t>t_{0}$ since there always exists a $t_{i}$ satisfying $t>t_{i} \geqq t_{0}$. Now $V_{t}(p)=\bigcap_{\varepsilon>0} U_{t+\varepsilon}(p)$ and if $q \in V_{t}(p)$ then $q \in U_{t+\varepsilon}(p), \varepsilon>0$. Since $q \in V_{i}(p)$ for all $t>t_{0}$ this implies $q \in U_{t_{0}+\varepsilon}(p) \supset U_{t_{0}+\varepsilon}(p)$, for all $\varepsilon>0$. Therefore

$$
q \in \bigcap_{\varepsilon>0} U_{t_{0}+\varepsilon}(p)=V_{t_{0}}(p)
$$

LEMmA 3. If $p_{2} \in V_{a}\left(p_{1}\right)$, then $a \geqq \rho\left(p_{1}, p_{2}\right)$.

Proof. Follows directly from definitions.

Using the notation $I$ for the unit interval, the following definition for an arc will be used. A set $S$ is an arc with endpoints $p_{1}, p_{2}$ in case there exists a continuous $1-1$ mapping of $I$ onto $S$ such that $f(0)=p_{1}, f(1)=p_{2}$. If $S \equiv f[I]$ is an arc, its length is defined as

$$
l(S)=\sup _{\left\{x_{i}\right\}} \sum_{i=0}^{N-1} \rho\left(f\left(x_{i}\right), f\left(x_{i+1}\right)\right)
$$


where $\left\{x_{i} \mid 0=x_{0}<x_{1}<\cdots<x_{N}=1\right\}$ is any partition of $I$. If $l(S)<\infty$, then $S$ has finite length; otherwise, the length of $S$ is infinite.

The following theorems indicate some of the relationships between arcs connecting $p_{1}$ to $p_{2}$ and the sets defined earlier.

THEOREM 1. If $A$ is an arc of length $c<\infty$ connecting $p_{1}$ to $p_{2}$, then $p_{2} \in V_{c}\left(p_{1}\right)$.

Proof. For each $j=1,2, \cdots$, choose points $q_{0} \equiv p_{1}, q_{1}, q_{2}, \cdots, q_{j-1}, q_{j} \equiv p_{2}$ on $A$ such that the arclength intercepted between successive points is $c / j$. For any $\varepsilon>0, \quad q_{i} \in T_{(c+\varepsilon) / j}\left(q_{i-1}\right), \quad(i=1,2, \cdots, j)$; this implies that $q_{i} \in T_{(c+\varepsilon) / j}^{(i)}\left(q_{0}\right)$, $(i=1,2, \cdots, j)$ and thus $p_{2} \equiv q_{j} \in T_{(c+\varepsilon) / j}^{(j)}\left(p_{1}\right)(\varepsilon>0, j=1,2, \cdots)$. Hence:

and in turn

$$
p_{2} \in \bigcap_{j=1} T_{(c+\varepsilon) / j}^{(j)}\left(p_{1}\right)=U_{c+\varepsilon}\left(p_{1}\right)
$$

$$
p_{2} \in \bigcap_{\varepsilon>0} U_{c+\varepsilon}\left(p_{1}\right)=V_{c}\left(p_{1}\right)
$$

REMARK 1. The stronger statement $p_{2} \in U_{c}\left(p_{1}\right)$ cannot be made, as the example of a straight line of length $c$ with $p_{1}$ and $p_{2}$ as endpoints shows.

THEOREM 2. If $A$ is an arc of length $c<\infty$ connecting its endpoints $p_{1}$ and $p_{2}$, then every point $q \subseteq A$ lies in $V_{a}\left(p_{1}\right) \cap V_{c-a}\left(p_{2}\right)$ for some $0 \leqq a \leqq c$.

Proof. Let the length of the subarc of $A$ from $p_{1}$ to $q$ be $a$; then, that from $q$ to $p$ is of length $c-a$. By Theorem $1, q \in V_{a}\left(p_{1}\right)$ and $q \in V_{c-a}\left(p_{2}\right)$.

ReMARK 2. The following example shows that $V_{a}\left(p_{1}\right) \cap V_{c-a}\left(p_{2}\right)$ does not always reduce to a unique point even when there is a unique minimizing arc joining $p_{1}$ to $p_{2}$. The space is the plane referred to polar coordinates where the set $r<1,(0 \leqq \theta<\pi), r \leqq 1,(\pi \leqq \theta<2 \pi)$ has been removed, $V_{\sqrt{ } 3 / 2}(2, \pi)$ $\cap V_{\pi / 3+(3 / 2) \sqrt{ } 3}(2,0)$ consists of two points.

REMARK 3. The set $V_{c}(p)$ need not be closed relative to the space. As an example let the space consist of the line segments $(0,0)$ to $(1,0) ;(0,1 / 2)$ to $(0,1)$; and $(1 / n, 0)$ to $(1 / n, 1), n=1,2, \cdots$. Then $V_{2}(1,0)$ does not contain the line segment $(0,1 / 2)$ to $(0,1)$.

REMARK 4. The set $V_{c}(p)$ need not be connected. As an example let $p_{1} \equiv(0,1), p_{2} \equiv(2,0)$. The space, Figure 2 , consists of the line joining $p_{1}$ to $(2,1)$ and $p_{2}$ to $(0,0)$ as well as a number of zig-zag lines each of length 10 joining the first of these segments to the second. The first such zig-zag line joins $(1 / 4,0)$ to $(1 / 4,1)$ and lies entirely in the rectangle bounded above and below by the two line segments, and on the sides by the verticals through $x=0$ and $x=1 / 2$. The second zig-zag line joins $(9 / 8,0)$ to $(9 / 8,1)$ and lies in the verticals through $x=1, x=5 / 4$. In general, the $n$th zig-zag line, $(n=0,1, \cdots)$ joins $\left(\left(2^{n+3}-7\right) / 2^{n+2}, 0\right)$ 


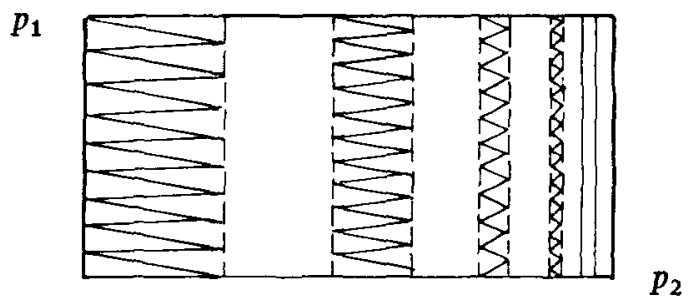

Figure 2.

to $\left(\left(2^{n+3}-7\right) / 2^{n+2}, 1\right)$ and lies in the rectangle bounded above and below by the horizontal line segments, and on the sides by the verticals through $x=\left(2^{n}-1\right) / 2^{n-1}$, $x=\left(2^{n+2}-3\right) / 2^{n+1}$. For this example $V_{3}\left(p_{1}\right)$ consists of the upper horizontal line segment, a portion of each zig-zag line of length $3-\left(2^{n+3}-7\right) / 2^{n+2}$ connected to this line segment, and the point $p_{2}$. The point $p_{2}$ is not connected to the rest of $V_{3}\left(p_{1}\right)$.

To better understand the subsequent discussions, one may observe that on the surface of a sphere the distance between two points can be defined in two obvious ways: as the straight-line distance in the metric $E^{(3)}$ from one of the points to the other, or, as the distance along the great circle minimal paths joining the two points. In this example, both are metrics. However, if the original space is a disconnected subset of $E^{(3)}$, only the former is a metric as the minimal arc length functional will not be defined for any pair of points not arcwise connected. The purpose in defining the $V_{c}(p)$ is to permit the definition of the function:

$$
\sigma\left(p_{1}, p_{2}\right)=\inf \left\{c \mid p_{2} \in V_{c}\left(p_{1}\right)\right\}
$$

which will be seen to be closely allied to the minimal arc length functional and also to have essentially the properties of a metric wherever it is defined as a finite number.

THEOREM 3. (a) $\sigma\left(p_{1}, p_{2}\right) \geqq 0$ and $\sigma\left(p_{1}, p_{2}\right)=0$ if and only if $p_{1}=p_{2}$;

(b) $\sigma\left(p_{1}, p_{2}\right)=\sigma\left(p_{2}, p_{1}\right)$ when either quantity is defined;

(c) if $\sigma\left(p_{1}, p_{2}\right)$ and $\sigma\left(p_{2}, p_{3}\right)$ are defined, then $\sigma\left(p_{1}, p_{3}\right) \leqq \sigma\left(p_{1}, p_{2}\right)+\sigma\left(p_{2}, p_{3}\right)$.

Proof. (a) Since $p_{1} \in V_{a}\left(p_{1}\right)$ for all $a>0$ it follows at once from the definition of $\sigma$ that $\sigma\left(p_{1}, p_{1}\right)=0$. Also, it follows from the definition that $\sigma\left(p_{1}, p_{2}\right)>0$, if $p_{1} \neq p_{2}$. (b) Let $c>\sigma\left(p_{1}, p_{2}\right)$. Since $p_{2} \in V_{c}\left(p_{1}\right)=\bigcap_{e}>0 U_{c+\varepsilon}\left(p_{1}\right)=$ $\bigcap_{\varepsilon>0} \bigcap_{j=1}^{\infty} T_{(c+\varepsilon) / j}^{(j)}\left(p_{1}\right)$ it follows $p_{2} \in T_{(c+e) / j}^{(j)}\left(p_{1}\right)$ for all $\varepsilon$ and $j$.

Choose $\varepsilon, j$ arbitrarily; we will show that $p_{1} \in T_{(c+\varepsilon) / j}^{(j)}\left(p_{2}\right)$. With $\varepsilon_{1}=\frac{1}{2} \varepsilon$ choose $j_{1}$ such that $\left(c+\varepsilon_{1}\right) / j_{1}<\varepsilon / 2 j$ and $j_{1}=n j$ where $n$ is some integer. By the definition of $T_{\left(c+\varepsilon_{1}\right) / j_{1}}(q)$ and $T_{\left(c+\varepsilon_{1}\right) / j_{1}}^{\left(j_{1}\right)}\left(p_{1}\right)$ there is a sequence of points $\left\{p_{1}=q_{1}, q_{2}, \cdots, q_{j_{1}+1}=p_{2}\right\}$ such that $q_{v+1} \in T_{\left(c+\varepsilon_{1}\right) / j_{1}}\left(q_{v}\right), \quad\left(v=1,2, \cdots, j_{1}\right)$. 
We define $q_{k+1}^{\prime} \equiv q_{J_{1}+1-k n}(k=0,1, \cdots, j)$ and note that $q_{1}^{\prime} \equiv p_{2}, q_{j+1}^{\prime} \equiv p_{1}$. Since $\rho\left(q_{k}^{\prime}, q_{k+1}^{\prime}\right) \leqq n\left(c+\varepsilon_{1}\right) / j_{1}$, therefore $q_{k+1}^{\prime} \in S_{n\left(c+\varepsilon_{1}\right) / j_{1}}\left(q_{k}^{\prime}\right)=S_{(\mathfrak{c}+\varepsilon / 2) / j}\left(q_{k}^{\prime}\right)$. Also,

so that:

$$
\rho\left(q_{k}^{\prime},\left(q_{k+1}^{\prime}\right)+\frac{c+\varepsilon_{1}}{j_{2}} \leqq\left(\frac{c+\varepsilon / 2}{j}\right)+\frac{\varepsilon}{2 j}=\frac{c+\varepsilon}{j}\right.
$$

and similarly

$$
S_{\left(c+\varepsilon_{1}\right) / j_{1}}\left(q_{k+1}^{\prime}\right) \subset S_{(c+\varepsilon) / j}\left(q_{k}^{\prime}\right),
$$

$$
S_{\left(c+\varepsilon_{1}\right) / j_{1}}\left(q_{j_{1}+1-k n+\alpha}\right) \subset S_{(c+\varepsilon) / j}\left(q_{k}^{\prime}\right),
$$

$(\alpha=0,1, \cdots, n)$. Thus $T_{\left(c+\varepsilon_{1}\right) / j_{1}}\left(q_{j_{1}+1-k n+\alpha}\right) \subset S_{(c+\varepsilon) / j}\left(q_{k}^{\prime}\right)$ and using the property of connected sets that if $q_{v}$ is in the component of $q_{v+1}$ and $q_{v-1}$, then $q_{v-1}$ is the component of $q_{v+1}$ it follows that $T_{\left(c+\varepsilon_{1}\right) / j_{1}}\left(q_{j_{1}+1-k n+\alpha}\right) \subset T_{(c+s) / j}\left(q_{k}^{\prime}\right)$ and in particular $T_{\left(c+z_{1}\right) i j_{1}}\left(q_{k+1}^{\prime}\right) \subset T_{(c+z) / j}\left(q_{k}^{\prime}\right)$. Therefore

$p_{1} \in T_{(c+\varepsilon) / j}\left(q_{j}^{\prime}\right) \subset T_{(c+\varepsilon) / j}^{(2)}\left(q_{j-1}\right) \subset T_{(c+\varepsilon) / j}^{(3)}\left(q_{j-2}^{\prime}\right) \subset \cdots \subset T_{(c+\varepsilon) / j}^{(j)}\left(q_{1}^{\prime}\right)=T_{(c+\varepsilon) / j}^{(j)}\left(p_{2}\right)$

as stated.

By the preceding paragraph $p_{1} \in T_{(c+\varepsilon) / j}^{(j)}\left(p_{2}\right)$ for all $\varepsilon, j$ and therefore $p_{1} \in \bigcap_{j=1}^{\infty} T_{(c+\varepsilon) / j}^{(j)}\left(p_{2}\right)=U_{c+\varepsilon}\left(p_{2}\right)$ for all $\varepsilon$; or $p_{1} \in \bigcap_{\varepsilon>0} U_{c+\varepsilon}\left(p_{2}\right)=V_{c}\left(p_{2}\right)$, so that $\sigma\left(p_{2}, p_{1}\right) \leqq c$. Therefore $\sigma\left(p_{2}, p_{1}\right) \leqq \inf \left\{c \mid p_{2} \in V_{\mathrm{c}}\left(p_{1}\right)\right\}=\sigma\left(p_{1}, p_{2}\right)$. The argument may be reversed to show that $\sigma\left(p_{1}, p_{2}\right) \leqq \sigma\left(p_{2}, p_{1}\right)$.

(c) It is a trivial matter to show from the definitions that if $p_{2} \in V_{a}\left(p_{1}\right)$, $p_{3} \in V_{b}\left(p_{2}\right)$ then $p_{3} \in V_{a+b}\left(p_{1}\right)$ and from this

$$
\sigma\left(p_{1}, p_{3}\right) \leqq \sigma\left(p_{1}, p_{2}\right)+\sigma\left(p_{2}, p_{3}\right) .
$$

LEMMA 4. If $\sigma\left(p_{1}, p_{2}\right)$ is defined, then $\rho\left(p_{1}, p_{2}\right) \leqq \sigma\left(p_{1}, p_{2}\right)$.

Proof. For every $\varepsilon>0, p_{2} \in V_{\sigma\left(p_{1}, p_{2}\right)+\varepsilon}\left(p_{1}\right)$; therefore by Lemma 3 , $\rho\left(p_{1}, p_{2}\right) \leqq \sigma\left(p_{1}, p_{2}\right)+\varepsilon$ and the statement follows.

The definitions introduced previously may now be related to the problem of constructing the path of minimal length joining two points $p_{1}, p_{2}$ in the space $S$. For any value of the number $a, 0 \leqq a \leqq \sigma\left(p_{1}, p_{2}\right)$, define

$$
C(a) \equiv V_{a}\left(p_{1}\right) \cap V_{\sigma\left(p_{1}, p_{2}\right)-a}\left(p_{2}\right)
$$

and set up the correspondence $R:[0,1] \rightarrow \bigcup_{a \in\left[0, \sigma\left(p_{1}, p_{2}\right)\right]} C(a)$ such that $R(x)$ $\equiv C\left(\sigma\left(p_{1}, p_{2}\right) x\right)$ for $x \in[0,1]$. It should be observed that for any given $x$, $C\left(\sigma\left(p_{1}, p_{2}\right) x\right)$ may be a null set, a singleton set, or a set consisting of more than one point; and that if $R(x)$ is singleton for each $x \in[0,1]$ then it is a singlevalued function.

THEOREM 4. If $r \in R(x)$ then $\sigma\left(p_{1}, r\right)+\sigma\left(r, p_{2}\right)=\sigma\left(p_{1}, p_{2}\right)$.

Proof. There is a value $a, 0 \leqq a \leqq \sigma\left(p_{1}, p_{2}\right)$ such that $r \in V_{a}\left(p_{1}\right)$ 
$\cap V_{\sigma\left(p_{1}, p_{2}\right)-a}\left(p_{2}\right)$. By definition $\sigma\left(p_{1}, r\right) \leqq a$ and $\sigma\left(p_{2}, r\right) \leqq \sigma\left(p_{1}, p_{2}\right)-a$. By Theorem 3(b), $\sigma\left(p_{2}, r\right)=\sigma\left(r, p_{2}\right)$. Therefore:

$$
\sigma\left(p_{1}, r\right)+\sigma\left(r, p_{2}\right) \leqq a+\sigma\left(p_{1}, p_{2}\right)-a=\sigma\left(p_{1}, p_{2}\right) .
$$

Thus, $\sigma\left(p_{1}, r\right)+\sigma\left(r, p_{2}\right)=\sigma\left(p_{1}, p_{2}\right)$, by Theorem $3(\mathrm{c})$.

THEOREM 5. If $R(x)$ is an arc joining $p_{1}$ to $p_{2}$ then $l(R(x))=\sigma\left(p_{1}, p_{2}\right)$.

ProOF. Let $0=x_{0}<x_{1}<x_{2}<\cdots<x_{N}=1$ be any partition of $[0,1]$ and $r_{i}=R\left(x_{i}\right),(i=0,1, \cdots, N)$. Then $l(R(x))=\sup _{\left\{x_{i}\right\}} \Sigma_{i=0}^{N-1} \rho\left(r_{i}, r_{i+1}\right)$; but by Lemma 4 and Theorem 4,

$$
\sum_{i=0}^{N-1} \rho\left(r_{i}, r_{i+1}\right) \leqq \sum_{i=0}^{N-1} \sigma\left(r_{i}, r_{i+1}\right)=\sigma\left(r_{0}, r_{N}\right)=\sigma\left(p_{1}, p_{2}\right)
$$

so that $l(R(x)) \leqq \sigma\left(p_{1}, p_{2}\right)$. By Theorem $1, p_{2} \in V_{c}\left(p_{1}\right)$ when $c=l(R(x))$ and therefore by definition $\sigma\left(p_{1}, p_{2}\right) \leqq l(R(x))$. Thus, $\sigma\left(p_{1}, p_{2}\right)=l(R(x))$.

In order to simplify the statement of the next theorem we introduce the following definition. A subset of a metric space will be said to be boundedly sequentially compact (BSC) in case every bounded infinite sequence in the set has a limit point which is also in the set. It is to be noted that in the following theorem this condition is applied to the original metric space, and not necessarily on the arbitrary subset $S$ contained within the space.

THEOREM 6. Let the comprehending metric space $E$, be BSC. If $R(x)$ is a singleton set for each $x \in[0,1]$ and $R:[0,1] \rightarrow S$ is a closed function from $[0,1]$ into $E$, then $R(x), 0 \leqq x \leqq 1$ describes a minimizing path from $p_{1}$ to $p_{2}$ in $S$ which is unique up to parametric representation.

Proof. We will first show that $R(x)$ is continuous. Suppose that $t_{0}$ were a point of discontinuity of $R(x)$. There exists a sequence $\left\{x_{i} \mid i=1,2, \cdots\right\}$ with $x_{i} \rightarrow t_{0}$ for which $R\left(x_{i}\right)$ does not converge to $R\left(t_{0}\right)$. A subsequence $x_{i j}=t_{j}$, $j=1,2, \cdots$ either monotonically increasing or decreasing to $t_{0}$ can be extracted from $\left\{x_{i}\right\}$. Assume the former, as the proof in the latter case is analogous.

Consider the values $R\left(t_{j}\right)$. Each is contained in $V_{t_{j}}\left(p_{1}\right) \subset S_{t_{0}}\left(p_{1}\right)$ and is therefore bounded. The sequence $\left\{R\left(t_{0}\right)\right\}$ is bounded and must have a limit point in $E$. Suppose $P \neq R\left(t_{0}\right)$ is such a limit point. Extract from $\left\{t_{j}\right\}$ a subsequence: $t_{j k}=s_{k}, k=1,2, \cdots$ such that $R\left(s_{k}\right) \rightarrow P$.

Two cases are to be considered: Case (i), there exists an interval $\left[a, t_{0}\right]$ such that $R(x) \neq P$ for any $x \in\left[a, t_{0}\right]$; Case (ii), there exists a sequence of points $a_{1} \leqq a_{2} \leqq a_{3} \leqq \cdots$ such that $a_{i} \rightarrow t_{0}$ and $R\left(a_{i}\right) \equiv P$ for all $i$.

Case (i): Since $s_{k} \uparrow t_{0}$, from some point on, $s_{k} \in\left[a, t_{0}\right]$. Since $R(x)$ is a closed function and $R\left(s_{k}\right) \rightarrow P$, there must be a point $y \in\left[a, t_{0}\right]$ such that $R(y)=P$. This is a contradiction. 
Case (ii): Since $R\left(a_{i}\right)=P$ and $\left(1-a_{1}\right) \geqq\left(1-a_{2}\right) \geqq\left(1-a_{3}\right) \geqq \cdots$ and $\left(1-a_{i}\right) \rightarrow\left(1-t_{0}\right)$, and, moreover, $P \in V_{1-a_{i}}\left(p_{2}\right), i=1,2, \cdots$ it follows from Lemma 2 that $P \in V_{1-t_{0}}\left(p_{2}\right)$. Also, $P \in V_{a_{i}}\left(p_{1}\right) \subset V_{t_{0}}\left(p_{1}\right)$. Therefore $P \in V_{t_{0}}\left(p_{1}\right)$ $\cap V_{1-t_{0}}\left(p_{2}\right)$. By the singleton hypothesis $P$ cannot be different from $R\left(t_{0}\right)$. This is a contradiction.

The conclusion is therefore that any limit point of the sequence $\left\{R\left(t_{j}\right)\right\}$ is $R\left(t_{0}\right)$. Hence $R(x)$ is continuous at $x=t_{0}$, and thus on $0 \leqq x \leqq 1$.

$R(1)=V_{\sigma\left(p_{1}, p_{2}\right)}\left(p_{1}\right) \cap V_{0}\left(p_{2}\right)$ is singleton, and since $V_{0}\left(p_{2}\right)=p_{2}$ it follows $R(1)=p_{2}$. Similarly $R(0)=p_{1}$. Continuity and single-valuedness of $R(x)$ show that $R(x)$ describes an arc with endpoints $p_{1}, p_{2}$.

By Theorem $5, l(R(x))=\sigma\left(p_{1}, p_{2}\right)$. Suppose $F(x), 0 \leqq x \leqq 1$ were an arc with endpoints $p_{1}, p_{2}$ of length $d \leqq \sigma\left(p_{1}, p_{2}\right)$. If $d<\sigma\left(p_{1}, p_{2}\right)$ then $p_{2} \in V_{d}\left(p_{1}\right)$ by Theorem 2, contradicting $\sigma\left(p_{1}, p_{2}\right)=\inf \left\{c \mid p_{2} \in V_{c}\left(p_{1}\right)\right\}$. If $d=\sigma\left(p_{1}, p_{2}\right)$, let $q$ be any point of $F(x), 0 \leqq x \leqq 1$. Applying Theorem 2, suppose $a$ is any value such that $q \in V_{a}\left(p_{1}\right) \cap V_{\sigma\left(p_{1}, p_{2}\right)-a}\left(p_{2}\right)$. Again by Theorem 2 , let $q^{\prime}$ be the point of $R(x)$ such that $q^{\prime} \in V_{a}\left(p_{1}\right) \cap V_{\sigma\left(p_{1}, p_{2}\right)-a}\left(p_{2}\right)$. By the hypotheses of this theorem $q=q^{\prime}$. Thus, $F(x)$ differs from $R(x)$ at most by the parametric representation of the same pointset.

For the following theorem we generalize the previous notations for $C(a)$ and $R(x)$ to:

$$
\begin{aligned}
& C\left(a ; q_{1}, q_{2}\right) \equiv V_{a}\left(q_{1}\right) \cap V_{\sigma\left(q_{1}, q_{2}\right)-a}\left(q_{2}\right) \quad 0 \leqq a \leqq \sigma\left(q_{1}, q_{2}\right) \\
& R\left(x ; q_{1}, q_{2}\right)=C\left(x \sigma\left(q_{1}, q_{2}\right) ; q_{1}, q_{2}\right) \quad 0 \leqq x \leqq 1
\end{aligned}
$$

where $q_{1}, q_{2} \in S$.

THEOREM 7. If for every pair of points $q_{1}, q_{2} \in S$ for which $\sigma\left(q_{1}, q_{2}\right)$ is defined, $R\left(x ; q_{1}, q_{2}\right)$ is nonempty and $B S C(0 \leqq x \leqq 1)$, then every pair of points $p_{1}, p_{2} \in S$ for which $\sigma\left(p_{1}, p_{2}\right)$ is defined is connected by a minimizing $\operatorname{arc}$ in $S$.

Proof: For $x=0,1$ choose $p(x)=p_{1}, p_{2}$ respectively, and for $x=1 / 2$ choose $p(x)$ to be any point of $R\left(1 / 2 ; p_{1}, p_{2}\right)$. For $x=(2 k+1) / 2^{n+1}$ choose $p(x)$ any point of $R\left(1 / 2 ; p\left(k / 2^{n}\right), p\left((k+1) / 2^{n}\right), k=0,1, \cdots,\left(2^{n}-1\right) ; n=1,2, \cdots\right.$. Let $y$ be any other value of the parameter, and for a given $n$ let $k$ be that integer for which $k / 2^{n}<y<(k+1) / 2^{n}$. Since $\sigma\left(p\left(k / 2^{n}\right), p\left((k+1) / 2^{n}\right)\right.$ is defined, consider $\left.\left.\left.R) 2^{n} y-k ; p\right) k / 2^{n}\right), p\left((k+1) / 2^{n}\right)\right)$ and choose a point $r_{n}$ in this non-empty subset. We note that $r_{n} \in R\left(2^{n} y-k ; p\left(k / 2^{n}\right), p\left((k+1) / 2^{n}\right) \subset R(y, p(0), p(1))\right.$ and since the latter is BSC it contains at least one limit point for the sequence $\left\{r_{n}\right\}$. This we designate as $P(y)$. The limit point is unique for if $P^{\prime}(y)$ were a second limit point then, $\rho\left(P(y), P^{\prime}(y)\right)=t>0$. Since the $R\left(2^{n} y-k, p\left(k / 2^{n}\right)\right.$, $p\left((k+1) / 2^{n}\right) n=1,2, \cdots$ is a nested sequence when $k$ is defined as above for each $n$, and each set is itself BSC, it follows that $P(y)$ and $P^{\prime}(y)$ are elements of 
$R\left(2^{n} y-k ; p\left(k / 2^{n}\right), p\left((k+1) / 2^{n}\right)\right)$. But $R\left(2^{n} y-k ; p\left(k / 2^{n}\right), p\left((k+1) / 2^{n}\right)\right)$ $\subset S_{1 / 2^{n}}\left(p\left(k / 2^{n}\right)\right)$ for all $n$, and its associated $k$. When $n$ is sufficiently large, the diameter of the sphere is $1 / 2^{n-1}$ and is less than $t$.

To prove continuity, suppose $\left\{x_{n}\right\} \rightarrow y$, and let $\varepsilon>0$. Select any $x \in\left\{x_{n}\right\}$ such that $|x-y|<\varepsilon / 12 \sigma\left(p_{1}, p_{2}\right)$. There exists a $\delta_{1}, 0<\delta_{1}<\varepsilon / 12 \sigma\left(p_{1}, p_{2}\right)$, such that if $k, n$ satisfy $\left|k / 2^{n}-x\right|<\delta_{1}$ then $\rho\left(p\left(k / 2^{n}\right), p(x)\right)<\varepsilon / 4$; also, there exists a $\delta_{2}, 0<\delta_{2}<\varepsilon / 12 \sigma\left(p_{1}, p_{2}\right)$ such that if $k^{\prime}, n^{\prime}$ satisfy $\left|k^{\prime} / 2^{n^{\prime}}-y\right|<\delta_{2}$ then $\rho\left(p\left(k^{\prime} / 2^{n^{\prime}}\right), p(y)\right)<\varepsilon / 4$. We note

$$
\left|\frac{k}{2^{n}}-\frac{k^{\prime}}{2^{n}}\right| \leqq\left|\frac{k}{2^{n}}-x\right|+|x-y|+\left|y-\frac{k^{\prime}}{2^{n}}\right| \leqq \varepsilon / 4 \sigma\left(p_{1}, p_{2}\right)
$$

and

$$
\rho\left(p\left(k / 2^{n}\right), p\left(k^{\prime} / 2^{n^{\prime}}\right)\right) \leqq \sigma\left(p_{1}, p_{2}\right)\left|\frac{k}{2^{n}}-\frac{k^{\prime}}{2^{n^{\prime}}}\right| \leqq \sigma\left(p_{1}, p_{2}\right) \varepsilon / 4 \sigma\left(p_{1}, p_{2}\right)=\varepsilon / 4 .
$$

Therefore, for $|x-y|<\varepsilon / 12 \sigma\left(p_{1}, p_{2}\right)$ we have:

$$
\left.\left.\rho(p(x), p(y)) \leqq \rho\left(p(x), p\left(k / 2^{n}\right)\right)+\rho\left(p\left(k / 2^{n}\right), p\right) k^{\prime} / 2^{n^{\prime}}\right)\right)+\rho\left(p\left(k^{\prime} / 2^{n^{\prime}}, p(y)\right)<3 \varepsilon / 4 .\right.
$$

Thus, we see that $p(x)$ is an arc in $S$ joining $p_{1}$ to $p_{2}$.

To prove minimality we observe that $p(x)$ is a singleton subset of $R\left(x ; p_{1}, p_{2}\right)$ for each $0 \leqq x \leqq 1$. Applying the same arguments to $p(x)$ as were used in the proofs of Theorems 4 and 5 it follows that $l(p(x))=p\left(p_{1}, p_{2}\right)$. Suppose $F(x)$, $0 \leqq x \leqq 1$ were an arc with endpoints $p_{1}, p_{2}$ of length $d$. If $d<\sigma\left(p_{1}, p_{2}\right)$ then $p_{2} \in V_{d}\left(p_{1}\right)$ by Theorem 2, contradicting $\sigma\left(p_{1}, p_{2}\right)=\inf \left\{c \mid p_{2} \in V_{c}\left(p_{1}\right)\right\}$.

COROLLARY: Under the conditions of the previous theorem there exists a unique minimizing arc up to parametric representation joining $p_{1}$ to $p_{2}$ if and only if $R\left(x ; p_{1}, p_{2}\right)$ is a singleton set for every value of $x$ on $I$.

Proof. We have seen that $p(x), 0 \leqq x \leqq 1$, is a minimizing arc joining $p_{1}$ to $p_{2}$. If $F(y)$ were a second minimizing arc, let it be referred to arc length measured from $p_{1}$ as parameter. Let $x=y / \sigma\left(p_{1}, p_{2}\right)$. By Theorem $1, F\left(x \sigma\left(p_{1}, p_{2}\right)\right)$ is an element of both $V_{x \sigma\left(p_{1}, p_{2}\right)}\left(p_{1}\right)$ and $V_{(1-x) \sigma\left(p_{1}, p_{2}\right)}\left(p_{2}\right)$ and consequently of $R\left(x ; p_{1}, p_{2}\right)$. But $p(x) \in R\left(x ; p_{1}, p_{2}\right)$. Thus if $R\left(x ; p_{1}, p_{2}\right)$ is singleton $p(x)$ $\equiv F\left(x \sigma\left(p_{1}, p_{2}\right)\right)$; and conversely if $p(x)$ differs from $F\left(x \sigma\left(p_{1}, p_{2}\right)\right)$ for any value of $x$, then $R\left(x ; p_{1}, p_{2}\right)$ is not singleton for that value of $x$.

In conclusion, we make the following remarks. The conditions of Theorem 7 give sufficient conditions for $S$ to be a globally geodesic space - that is to say, a space in which every pair of points are arcwise connected by arcs of finite minimal length. For if $q_{1}, q_{2}$ are two points of $S$ which are not arcwise connected, then $\sigma\left(q_{1}, q_{2}\right)$ is not defined. If they are connected only by arcs of unbounded length, again $\sigma\left(q_{1}, q_{2}\right)$ is undefined. If there is a plurality of minimizing arcs joining $q_{1}$ to $q_{2}$ then $R\left(x ; q_{1}, q_{2}\right)$ is not singleton for some value of $x$. Finally, 
when $R\left(x ; q_{1}, q_{2}\right)$ is more than singleton and the other hypotheses are also satisfied, then $q_{1}$ and $q_{2}$ are conjugate points in the sense that they are joined by more than one arc of equal minimal length.

Regrettably, it may be noted that the conditions are not necessary as the example of an open hemisphere in $E^{(3)}$ together with two antipodal points $q_{1}, q_{2}$ adjoined on the boundary shows. Here $q_{1}$ and $q_{2}$ are joined by a family of great circle minimizing arcs on the hemisphere but $R\left(x ; q_{1}, q_{2}\right)$ is not BSC for $0<x<1$.

\section{References}

H. W. Milnes and S. H. Hildebrand (1970), 'Non-isolated minimising arcs', SIAM J. Appl. Math. 18, 139-149.

H. W. Milnes and S. K. Hildebrand (1972), 'Mappings that remove singularities', Texas J. Science 24, 183-190.

H. W. Milnes and S. K. Hildebrand (1972), 'Maxima and minima of functions and functionals', Studia Sci. Math. Hungar. 7, 249-256.

H. W. Milnes and S. K. Hildebrand (submitted), 'Endpoint conditions for the problem of extremizing a non-parametric integral in the plane'.

Texas Tech University

Lubbock, Texas 79409

U. S. A.

and

H-M Consultants

Lubbock, Texas 79409

U. S. A. 